preclinical biomarker validation compared to antibody-based assays.

SomaLogic customers will soon be able to submit proteins for a service to generate a specific SOMAmer-but at the steep cost of handing over the intellectual property rights to the molecule both for use as a potential therapeutic and for expanding SOMAscan's repertoire. SomaLogic expects to reach 3,000 SOMAmers by the end of this year and 5,000 by the end of 2015, with the eventual goal of covering the roughly 20,000 proteins in the human proteome, says Hewett.

The technology does have its limitations, of course. Baird notes that the proprietary nature of SOMAmers means that researchers don't always know exactly which parts of a protein the SOMAmer binds to. To date, the three-dimensional structures of only two SOMAmer-protein pairs have been published $^{6,7}$. As such, it's not always clear what's being tracked-the fully functional protein or a cleaved snippet.

Wagers notes that SOMAscan is not yet the 'go-to' answer for all proteomics questionsafter all, it currently only covers about $6 \%$ of the human proteome today. But, she says, it's an amazingly powerful technology, especially for challenging protein identifications: "If they happen to have an aptamer to your needle in the haystack, then it's perfect."

Kendall Powell

1. Loffredo, F.S. et al. Cell 153, 828-839 (2013).

2. Vaught, J.D. et al. J. Am. Chem. Soc. 132, 4141-4151 (2010).

3. Gold, L. et al. PLOS ONE doi:10.1371/journal. pone.0015004 (7 December 2010)

4. Zhang, B. et al. Nature 513, 382-387 (2014).

5. Shi, T. et al. Proc. Natl. Acad. Sci. USA 109, 15395 15400, (2012)

6. Davies, D.R. et al. Proc. Natl. Acad. Sci. USA 109 19971-19976 (2012).

7. Gelinas, A.D. et al. J. Biol. Chem. 289, 8720-8734 (2014).

\title{
Connectivity webs from HIV sequences could inform counseling
}

For those living with HIV, viral load-the amount of viral RNA circulating in the bloodand the number of $\mathrm{CD}^{+} \mathrm{T}$ cells per cubic millimeter of blood are key to knowing how well antiretroviral therapy (ART) is working and how high their risk of transmitting the virus may be. But those two numbers are not the whole story. Sexual behavior and drug use, among other variables, also contribute to transmission risk. A study published this summer traced specific sequences in the HIV genome within networks of HIV-positive individuals to find out whether genetic data could be used to generate another estimate of transmission risk (PLoS ONE 9, e98443, 2014).

Susan Little, an infectious disease specialist at the University of California, San Diego (UCSD) School of Medicine led a retrospective analysis of HIV sequence data collected in San Diego clinics through two screening programs called "Early Test" and "Lead the Way", an ongoing campaign funded mainly by the US National Institutes of Health that offers free universal HIV testing. Between 1996 and 2011, Little's group collected blood samples from 478 of the people diagnosed through the programs and 170 more from their HIV-positive social and sexual contacts. Her group searched for mutations in a viral gene called pol, which is routinely sequenced in US clinics because it commonly mutates, causing drug resistance. "We can use tiny differences between HIV sequences to map the evolution of the virus as it spreads," Little says.

The UCSD researchers grouped HIV-positive people with similar pol sequences into clusters and, based on characteristics of those clusters, generated transmission network scores (TNSs) for each individual. The TNS is a measure of connectivity within a given network. As an example, Little says that her group can tell that there is a growing network of HIV-positive individuals among Hispanic men between the ages of 15 and 35 in southern San Diego. If someone of that demographic has a high TNS, his virus was probably transmitted very often within that group.

However, it doesn't prove that he contracted the virus from within that group. "You'll never know who gave the virus to whom," comments Ann Dennis, who studies HIV transmission at the University of North Carolina at Chapel Hill School of Medicine.

\section{All about networking}

All new HIV cases in the US are reported to local health departments, which in turn report de-identified information to the Centers for Disease Control and Prevention (CDC). In many states, health departments contact partners who were potentially exposed, and often a disease intervention specialist (DIS) will interview the diagnosed individual and build a potential transmission network by hand, a time-consuming and laborious process subject to many biases. "By hand' is a good way to put it because not everyone even keeps digital records," says Erik Volz, a population biology and infectious disease researcher at Imperial College London.

Networks built from genetic data may more accurately identify nodes of transmission and high-risk groups who will most benefit from intervention efforts. "Our simulation would suggest that if we treat people with the highest TNS scores, we'll have the greatest effect on interrupting transmission," she says. Although she stresses that everyone with HIV should be on therapy, TNSs can help with allocating public health resources such as preexposure prophylaxis, frequent testing or prevention education and counseling.

But these networks come with caveats. Those

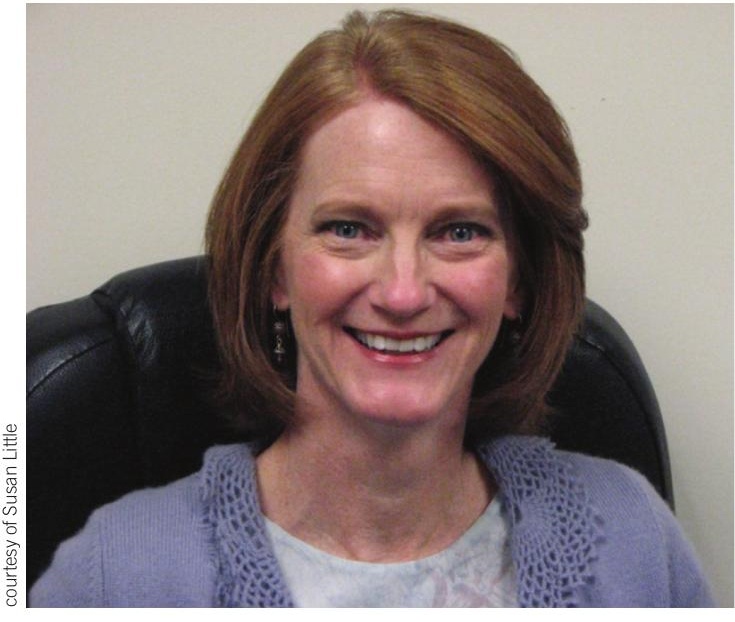

Linked in: Little studies viral networks.

whose sequencing was done by a different company or an out-of-state clinic would be excluded from a county- or state-based transmission network model. As such, Volz says genetic data should be complementary to DIS efforts.

Little says her results still need to be replicated independently with a different study population. Eventually, she says, the TNS approach could be a useful metric reported along with viral load and CD4 count to make individuals aware of their transmission potential and inform treatment and behavioral decisions. She is now working on a prospective study to follow HIVpositive individuals who are part of known genetic transmission networks longitudinally after they get on ART. If the TNS is accurate, she thinks treating those with higher scores will interfere with transmission networks to a greater degree than treating those with low scores. "That's where the field needs to move-into prospective use of sequence data," says Dennis. Amanda B Keener 